\title{
Mixed results for novel CD20-targeted antibody
}

The results of Phase III trials of the CD20-targeted human monoclonal antibody $(\mathrm{mAb})$ ofatumumab in rheumatoid arthritis and non-Hodgkin's lymphoma (NHL) dampen hopes on the potential of the drug to achieve the success of the pioneering CD20-targeted mAb rituximab (Rituxan/ MabThera; Genentech/Roche/Biogen Idec).

Rituximab, a chimeric murine-human $\mathrm{mAb}$, was first approved in 1997 for the treatment of patients with relapsed or refractory B cell NHL that is CD20-positive. Since then, additional approvals for NHL, rheumatoid arthritis and chronic lymphocytic leukaemia (CLL) have made rituximab a major commercial success, with Roche reporting sales of $\sim 5.9$ billion Swiss francs in 2008.

The expression of CD20 on the surface of $\mathrm{B}$ cells provided the rationale for developing rituximab for B cell malignancies such as NHL. "Anti-CD20 antibodies have demonstrated the ability to kill B lymphocytes in animal models and cause malignant cell lysis by activating complement- and antibody-dependent cellular toxicity (ADCC)," explains Michael Keating, Professor of Medicine, MD Anderson Cancer Center, Texas, USA.

$B$ cells also have several roles that are thought to be important in autoimmune disorders such as rheumatoid arthritis: "They produce antibodies (including autoantibodies); they can activate antigen-specific T cells; and they produce a variety of cytokines," adds Josef Smolen, Professor of Medicine and Chairman of the Department of Rheumatology, University of Vienna, Austria. Although it is not clear which of these activities is the most important target for rituximab in rheumatoid arthritis, the initial success of this drug (Nature Rev. Immunol. 6, 394-403; 2006) has led to its evaluation in other autoimmune diseases.
Ofatumumab, a fully human mAb that is being developed by Genmab and GlaxoSmithKline (GSK), potentially offers the advantage of fewer adverse effects than rituximab. "While the early acute adverse events in the course of anti-CD20 therapy appear to be a consequence of cytokine release in the course of B cell depletion, later adverse events might be partly due to reactivity against the murine antigens of the chimeric molecule," explains Smolen.

An FDA decision on the approval of ofatumumab for CLL is anticipated at the end of October, following successful clinical trials reported in 2008. Now, the companies have reported top-line Phase III trial results in rheumatoid arthritis and NHL.

In the trial for rheumatoid arthritis - which involved 260 patients who had failed to respond to standard therapy with methotrexate - after 24 weeks, $50 \%$ of patients receiving ofatumumab met the primary efficacy end point, compared with $27 \%$ of patients in the placebo group. However, in the trial for NHL, which involved 116 patients who were refractory to rituximab, the overall response rate $(10 \%)$ was below that required to trigger a milestone payment from GSK.

Differences between ofatumumab and rituximab that might contribute to these results include the site at which the drugs bind to CD20. "Ofatumumab binds on a site which is closer to the cell membrane, and it is presumed to work more effectively by complement lysis rather than ADCC. It has also been shown to have a tighter binding with a slower off rate than other mAbs against CD20," says Keating. "The preclinical data suggest that it is more active than rituximab in cells that have a low number of CD20 molecules on the surface, which is characteristic of CLL but probably less important in NHL." 